\title{
An Open Labeled, Placebo Controlled Trial to Evaluate the Role of Probiotics- Bacillus subtilis HU58 and Bacillus coagulans SC208 0n Antibiotic Associated Diarrhoea In Humans
}

\author{
Mehta DS*1, De Souza A $^{2}$, Jadhav $S^{3}$ and Devale $\mathbf{M}^{4}$ \\ ${ }^{1}$ CEO, Synergia Life Sciences Pvt Ltd, Mumbai, India \\ ${ }^{2}$ Managing Director, Synergia Life Sciences Pvt Ltd, Mumbai, India \\ ${ }^{3}$ Med. Director, Synergia Life Sciences Pvt Ltd, Mumbai, India \\ ${ }^{4}$ Consulting Physician, Kokan Hospital, Mumbai, India \\ *Corresponding author: Dilip Mehta, CEO, Synergia Life Sciences Pvt Ltd, Mumbai, India
}

\section{ARTICLE INFO}

Received: 幽 August 07, 2020

Published: 慧 August 21, 2020

Citation: Dilip Mehta. An Open Labeled, Placebo Controlled Trial to Evaluate the Role of Probiotics- Bacillus subtilis HU58 and Bacillus Coagulans SC208 0n Antibiotic Associated Diarrhoea In Humans. Biomed J Sci \& Tech Res 29(4)-2020. BJSTR. MS.ID.004839.

Keywords: Probiotic Bacillus subtilis HU58; Bacillus coagulans SC208; Antibiotic Associated Diarrhea (AAD); Irritable Bowel Syndrome - Diarrhoea (IBS-D); Stool frequency and consistency; Bristol Stool Chart

\section{ABSTRACT}

Objective: To evaluate the activity and tolerability of a probiotic mix of Bacillus subtilis HU58 plus Bacillus coagulans SC208 and Bacillus coagulans SC208 alone compared with placebo in a group of 75 patients suffering from Antibiotic Associated Diarrhea (AAD).

Materials and Methods: An open labeled placebo-controlled trial was conducted for Bacillus subtilis HU58 + Bacillus coagulans SC208 in combination and Bacillus coagulans SC208 alone in patients suffering from AAD. Stool consistency was estimated according to Bristol Stool Chart (BSC) and was recorded at baseline, $3^{\text {rd }}$ day, $7^{\text {th }}$ day and on $15^{\text {th }}$ day along with other symptoms like abdominal pain, bloating and flatulence. All enrolled patients were orally administered either a cocktail of Bacillus subtilis HU58 + Bacillus coagulans SC208 ( 3 X 109 CFU/Cap), or Bacillus coagulans SC208 (2 X 109 CFU/ Cap) alone or placebo for 7 days. Blood investigations were done at baseline, $3^{\text {rd }}$ and $7^{\text {th }}$ day. A final follow up was done on $15^{\text {th }}$ day. This study was approved by an Independent Ethics Committee and registered with Clinical Trial Registry of India. A written Informed Consent Form was obtained from the patients before enrollment.

Results: Patients ( $\mathrm{n}=75)$ were randomly allocated in three groups; Group $\mathrm{A}(\mathrm{n}=25)$, received a combination of Bacillus subtilis HU58 + Bacillus coagulans SC208, Group $\mathrm{B}(\mathrm{n}=25)$ received Bacillus coagulans SC208 alone and Group C $(\mathrm{n}=25)$ received the placebo. The Bristol stool scale (Figure 1) which classifies human stool into seven categories was used to measure the consistency of stool during the study. Statistically significant improvement in stool consistency was seen in both the probiotic groups $(\mathrm{p}<0.0001)$ as compared to the placebo $(\mathrm{p}<0.0799)$. Stool consistency at baseline in all groups was at scale of 6-7. Improvement was observed in Group A (scale 3) and Group B (scale 5). No reduction of scores was observed in group $C$ (scale 7 ) till $15^{\text {th }}$ day. The other symptoms like pain in abdomen, bloating and flatulence were also reduced in both group A and group B as compared to group C.

Conclusion: Improvement in stool frequency and consistency along with symptoms like pain in abdomen, bloating and flatulence was found in individuals in group A and group B. Data of patients in group A and group B was found to be statistically significant ( $p$ value $<0.0001$ ) compared to group C. It was also found that patients receiving the combination of Bacillus subtilis HU58 + Bacillus coagulans SC208 experienced much better relief in terms of stool frequency and consistency along with pain in abdomen, bloating and flatulence than patients receiving only the Bacillus coagulans SC208 strain. This comparison, however, needs to be further studied in detail by conducting double blind randomized study in larger sample size. 


\section{Introduction}

Antibiotics are one of the most over-prescribed drugs in modern medicine. The side effects of antibiotics are nausea, indigestion, vomiting, diarrhea, bloating and feeling of fullness, loss of appetite, stomach cramping and pain. Among these, diarrhea is the most common one and can range from mild to watery stools. 'Antibiotic-associated diarrhea is defined as otherwise unexplained diarrhea that occurs in association with the administration of antibiotics. The frequency and severity of AAD varies with different antibacterial agents and occurs in about $5-25 \%$ in patients and can occur with oral as well as parental antibiotics like ampicillin, amoxicillin, fluoroquinolones, cephalosporins, clarithromycin, erythromycin, and tetracycline [1]. About $9.6 \%$ of the hospitalized patients consuming antibiotics suffered from AAD [2]. Incidence of diarrhea in pediatrics in outpatient wards was found to be 6-75\%. In adults, the AAD occurs in 7-33 patients out of 100 patients taking antibiotics and even after the treatment for diarrhea, a relapse is seen in almost $20 \%$ of cases [1]. Probiotics reduce and shorten the duration of AAD which is shown in many studies [3-6]. AAD occurs due to the antimicrobial action which kills the normal gut microflora and enables the pathogenic organisms that induce diarrhea to overgrow.

One of the most notorious organisms which cause AAD in around $10-25 \%$ of cases is the spore forming anaerobe Clostridium difficile. Other species associated but to a lesser extent are $C$. perfringens, S. aureus, Candida spp, Klebsiella spp, and Salmonella spp [1]. Probiotics are often prescribed with the belief that the intake of healthy bacteria would correct the dysbiosis and recolonize the gut and thereby reduce AAD. There have been a wide range of studies with different strains of organisms and with patchy results [7]. Some of the commonly used organisms include Bacillus spp., Bifidobacterium spp., Clostridium butyricum, Lactobacilli spp., Lactococcus spp., Leuconostoccremoris, Saccharomyces spp., Streptococcus spp., Enterococcus spp., Pediococcus spp., Escherichia coli alone or in combination. In a probiotic preparation these organisms may be used as a single species or a mixture of multi species with variable spore counts [8]. The present study was undertaken to evaluate the efficacy of two probiotic preparations in patients with AAD.

\section{Materials and Methods}

\section{Study Design}

We conducted an open labeled, placebo-controlled trial in patients with AAD to compare the efficacy, safety and tolerability of the probiotic combination of Bacillus subtilis HU58 + Bacillus coagulans SC208, Bacillus coagulans SC208 alone and placebo. The study was conducted at Konkan Hospital, Mumbai with the approval of an Independent Ethics Committee and registered at Clinical Trial
Registry of India (CTRI) CTRI/2018/06/014439.

\section{Subjects}

The study was conducted at Department of Medicine of Kokan Hospital, Mumbai, India. Eighty patients (aged 18-65 years) who were on antibiotics and suffered from AAD were screened and selected after taking an informed consent. Out of 80 screened patients, 75 patients completed the study. All patients were randomized in group A, group B and group C as shown in Table 1.

Table 1: Number of patients in group A, group B and group C.

\begin{tabular}{|c|c|c|c|}
\hline & Group A & Group B & Group C \\
\hline $\begin{array}{c}\text { No. of patients } \\
\text { (n) }\end{array}$ & 25 & 25 & 25 \\
\hline Age (years) & $36(5.6)$ & $34(5.5)$ & $34(5.0)$ \\
\hline Gender & & & \\
\hline Men & 15 & 10 & 15 \\
\hline Women & 10 & 15 & 10 \\
\hline
\end{tabular}

Values are expressed in Mean (SD); Group A: Bacillus subtilis HU58 + Bacillus coagulans SC208, group B: Bacillus coagulans SC208 and group C: Placebo. SD: Standard Deviation.

\section{The Study Procedure and Assessments}

After proper history, examination and investigations seventyfive patients were selected as per inclusion criteria in the protocol. These patients complained of diarrhea due to intake of oral antibiotics. They also complained of pain in abdomen, sensation of bloating, flatulence along with diarrhea. Blood investigations viz. complete blood counts with ESR, PT-INR, lipid profile, S. Creatinine, homocysteine, urine analysis routine, stool routine, ECG, HIV, HCV, HBsAg, UPT, liver function tests were done at the base line On the $3^{\text {rd }}$ day and $7^{\text {th }}$ day all blood investigations were repeated except HIV, HCV, HBsAg. A detailed general and systemic examination was done at the baseline and at visits on $3^{\text {rd }}$ day, $7^{\text {th }}$ day and $15^{\text {th }}$ day. A predesigned case record form (approved by the Ethics Committee) was used. One capsule of either Bacillus subtilis HU58 + Bacillus coagulans SC208 in combination or Bacillus coagulans SC208 alone or placebo was prescribed to the patients daily for 7 days. The stool frequency was assessed with Bristol Stool Scale (Figure 1). BSC is a common tool and has been used in extensively to study the consistency of stool $[9,10]$. As per the Bristol stool scales, $1-2$ is constipated type of stool, 3-5 is normal stool and 6-7 is loose stool i.e., diarrhea [10]. The safety of probiotic Bacillus subtilis HU58 + Bacillus coagulans SC208 in combination and Bacillus coagulans SC208 was assessed by clinical tolerability, side effects and adverse events and by any change in the organ function tests. Improvement in stool consistency compared to baseline was noted to assess the therapeutic efficacy. Other beneficial and adverse effects which were observed during therapy were recorded. 


\section{Bristol Stool Chart}

\begin{tabular}{|l|l|}
\hline Type I & $\begin{array}{l}\text { Separate hard lumps, like nuts } \\
\text { (hard to pass) }\end{array}$ \\
\hline Type 2 & Sausage-shaped but lumpy \\
\hline Type 3 & Like a sausage but with cracks on \\
\hline its surface & Like a sausage or snake, smooth \\
\hline and soft
\end{tabular}

Figure 1: Bristol Stool Chart.

\section{Methods for Blood Investigations}

Complete blood counts were done by the PC 210 ERMA Blood Cell Counter. ESR was done by Wintrobe method. Liver function test were done by Biochemical method. Homocysteine was done by RIA/ ELISA/ CLIA method.

\section{Interventional Drug, Dosage and Compliance}

Synergia Life Sciences Pvt. Ltd. supplied Probiotic Bacillus subtilis HU58 + Bacillus coagulans SC208, Bacillus coagulans SC208 and identical placebo capsules (7 capsules/ bottle). Patients were supplied with the capsules at the time of enrollment.

\section{Results}

According to BSC, the stool consistency measured at baseline was same for all the three groups (scale 6-7). In group $A$, on third day, the stool consistency was improved (scale 5). A further improvement was observed in the stool consistency (scale 4) on $7^{\text {th }}$ day. At the time of follow up on $15^{\text {th }}$ day, the stool consistency further improved to scale 3. Patients were feeling better with Table 3: Statistical Analysis. the therapy. Other symptoms like pain in abdomen, sensation of bloating and flatulence reduced considerably. In group B, patients had stool consistency of scale 6 on third day. Improvement in stool consistency was observed (scale 4 ) at the end of $7^{\text {th }}$ day. During the follow up on $15^{\text {th }}$ day the stool consistency was observed at scale 5 . The other symptoms like pain in abdomen, sensation of bloating and flatulence also decreased. In group $\mathrm{C}$, the scale remained the same as baseline which was at 6-7. Improvement in stool consistency was not seen. Patients were not feeling better with the therapy. Table 2 shows the details about average stool consistency. The pain in abdomen was continuous with no relief. Also, the symptoms of flatulence and bloating sensation did not reduce.

Table 2: Average Bristol Stool Chart Scale data of Group A, Group B and Group C.

\begin{tabular}{|c|c|c|c|}
\hline & Group A & Group B & Group C \\
\hline No. of subjects & 25 & 25 & 25 \\
\hline Baseline & $6.60(0.50)$ & $6.64(0.49)$ & $6.48(0.51)$ \\
\hline End of 3rd day & $5.32(0.63)$ & $5.60(0.58)$ & $6.36(0.49)$ \\
\hline End of 7th day & $3.96(0.68)$ & $4.40(1.19)$ & $6.40(0.50)$ \\
\hline End of 15th day & $3.16(0.37)$ & $4.88(0.93)$ & $6.24(0.44)$ \\
\hline
\end{tabular}

Values are expressed in Mean (SD). Group A: Bacillus subtilis HU58 + Bacillus coagulans SC208, Group B: Bacillus coagulans SC208 and Group C: placebo. SD: Standard Deviation.

\section{Statistical Analysis}

Analysis of data was done for the two sets using student's unpaired $t$ test. In Group A the mean, standard deviation (SD), Standard Error of Mean (SEM) at baseline was found to be 6.63, $0.49,0.10$ respectively whereas on the 15 th day the mean, SD and SEM was found to be $3.17,0.38,0.08$ respectively. The unpaired $t$ test for this group was found to be statistically significant ( $\mathrm{p}$ value $<$ 0.0001). Mean, SD, SEM ingroup B for baseline was found to be $6.60,0.50,0.10$ respectively and by the end of $15^{\text {th }}$ day the mean, SD, SEM was found to be 3.16, $0.37,0.07$ respectively. The unpaired $\mathrm{t}$ test for this group was also found to be statistically significant ( $\mathrm{p}$ value $<0.0001$ ). Mean, SD, SEM in group C for baseline was found to be $6.48,0.51,0.10$ respectively and by the end of $15^{\text {th }}$ day the mean, SD, SEM was found to be 6.24, 0.44, 0.09 respectively. The data for group $C$ was not statistically significant ( $p$ value 0.0799 ) as compared to group A and group B. Table 3 shows the statistical analysis of group A, group B and group C.

\begin{tabular}{|c|c|c|c|c|c|c|}
\hline Diagnosis & \multicolumn{6}{|c|}{ Antibiotic Associated Diarrhoea (AAD) } \\
\hline Symptoms & \multicolumn{6}{|c|}{ Diarrhoea } \\
\hline Treatment & \multicolumn{2}{|c|}{ Group A } & \multicolumn{2}{|c|}{ Group B } & \multicolumn{2}{|c|}{ Group C } \\
\hline \multirow[t]{2}{*}{$\mathbf{N}$} & \multicolumn{2}{|c|}{25} & \multicolumn{2}{|c|}{25} & \multicolumn{2}{|c|}{25} \\
\hline & Baseline & $15^{\text {th }}$ day & Baseline & $15^{\text {th }}$ day & Baseline & $15^{\text {th }}$ day \\
\hline Bristol stool chart & 7 & 3 & 7 & 5 & 7 & 7 \\
\hline Mean & 6.6 & 3.16 & 6.64 & 4.88 & 6.48 & 6.24 \\
\hline SD & 0.5 & 0.37 & 0.49 & 0.93 & 0.51 & 0.44 \\
\hline
\end{tabular}




\begin{tabular}{|c|c|c|c|c|}
\hline SEM & 0.1 & 0.07 & 0.19 & 0.1 \\
\hline T & 27.542 & 8.3905 & 0.09 \\
\hline $\begin{array}{c}\text { standard error of } \\
\text { difference }\end{array}$ & 48 & 0.21 & 0.134 \\
\hline two-tailed P value & 0.125 & $<0.0001$ & 0.0799 \\
\hline Confidence interval & $<0.0001$ & $95 \%$ & $95 \%$ \\
\hline $\begin{array}{c}\text { 95\% confidence } \\
\text { interval of this } \\
\text { difference from }\end{array}$ & $95 \%$ & 1.34 to 2.18 & -0.03 to 0.51 \\
\hline
\end{tabular}

\section{Tolerability and Safety}

Probiotic capsules i.e. Bacillus subtilis HU58 + Bacillus coagulans SC208 and Bacillus coagulans SC208 were clinically well tolerated by all the patients. No adverse effects were observed in both the groups. All biochemistry investigations done at baseline, third day and seventh day were found to be within normal limits.

\section{Compliance}

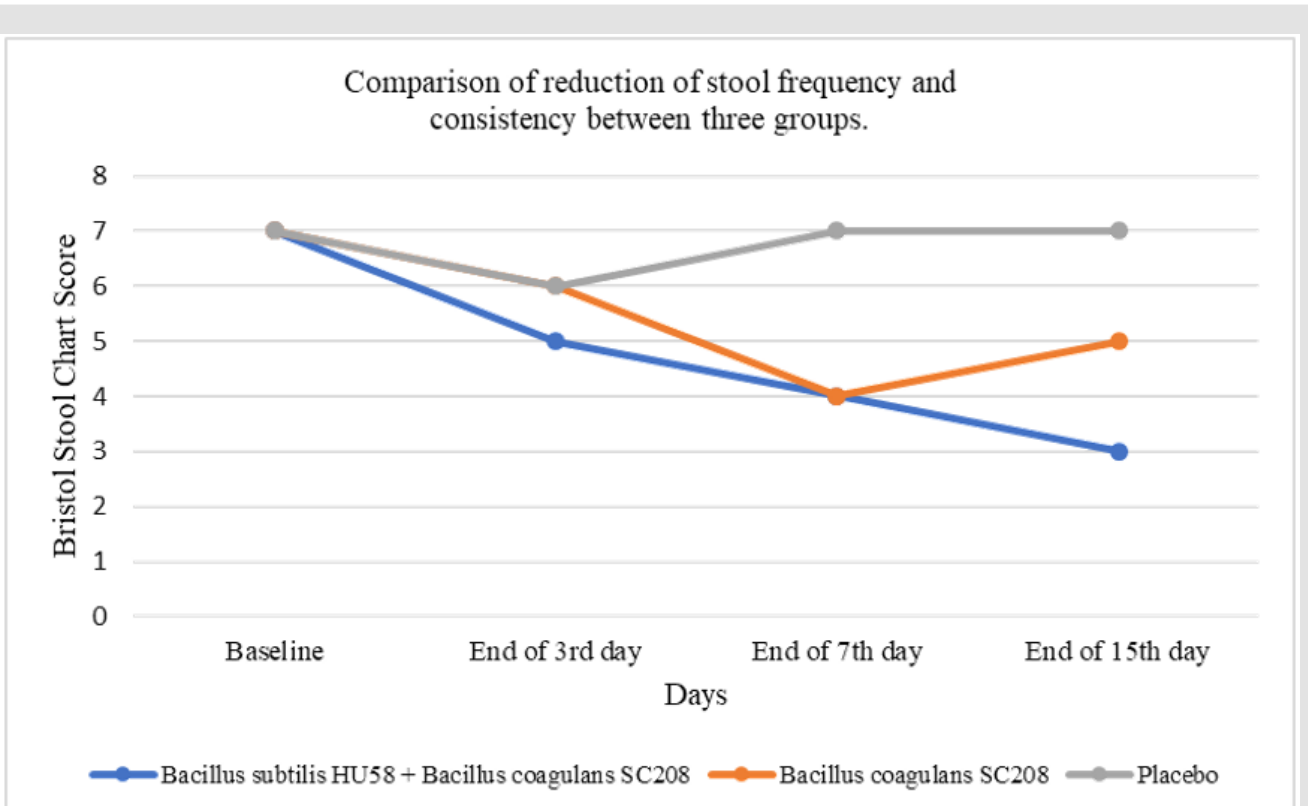

Figure 2: Comparison of stool consistency score as per Bristol Stool Scale for the patients receiving Bacillus coagulans SC208, Bacillus subtilis HU58 + Bacillus coagulans SC208 and Placebo from Baseline to Day 15.

Intake of capsules by patients which was monitored during the 7 days of trial showed that patients were regular in their intake of capsules. During follow up visits the patients were requested to bring the bottle containing the capsules and the capsules remaining were counted at the $3^{\text {rd }}$ and $7^{\text {th }}$ day. Compliance was judged during the follow up visits. Patients were said to be compliant if they had consumed minimum $80 \%$ of the total dispensed capsules (Figure 2).

\section{Discussion}

Bacillus species have been used as probiotics for at least 50 years. Of the species that have been most extensively examined are Bacillus subtilis and Bacillus coagulans. Bacillus subtilis strain HU58 has been studied extensively in detail by Prof. Simon Cutting and his group, at the Royal Holloway College, University London.
Bacillus coagulans is a lactic acid producing bacteria with typical characteristics of both Bacillus and Lactobacillus genera. Bacillus subtilis and Bacillus coagulans have been extensively studied at genetic and physiological levels [11]. Bacillus subtilis and Bacillus coagulans are more stable in acidic stomach environment, can grow and sporulate with high efficiency in anaerobic conditions in the gastrointestinal tract, form biofilms which enhance gut colonization and produce surfactants which enhances the gut adhesion $[12,13]$. Oral supplementation of these two probiotics provides useful supply of beneficial bacteria for gut health, aiding nutrition and potentially stimulating the immune system.

Recently an open labeled study was conducted by Dound YA et al in 18 healthy subjects who were administered Bacillus subtilis HU58 ( 2 X $\left.10^{9} \mathrm{CFU}\right)$ once a day orally for 8 weeks. At the end of $8^{\text {th }}$ weeks, it was observed that there was reduction in IL- 6 by $45 \%$ and 
TNF- $\alpha$ by 55\% [14]. Further, in an open labeled study conducted by Mehta DS et al in 60 patients suffering from AAD, Bacillus subtilis HU58 $\left(2 \times 10^{9} \mathrm{CFU}\right)$ daily for 7 days showed a reduced incidence of AAD in comparison with placebo [15]. The gut microbiota controls essential human functions like digestion, energy metabolism, and inflammation by modifying multiple endocrines, neural, and immune pathways of the host [16]. The interaction of microbes in intestinal tissue where the growth of microbes takes place in the lumen contributes to digestion of food and chemical milieu of the gut [17]. The non-digestible carbohydrates produce primary endproduct such as short chain fatty acids there-by providing health benefits [18]. Administration of antibiotics even for short periods, disturbs the normal microbiota of the gut leading to a dysbiosis state which aggravates the disease [16]. Antibiotic administration triggers changes in enteric bacterial flora and a reduction in flora of fecal anaerobes due to which leads to alteration in carbohydrate metabolism and digestion followed by a concurrent decrease in absorption of short chain fatty acids causing osmotic diarrhea [19].

Probiotics administration helps to improve the microbial imbalance of the host. They have the ability to fight against infections of mucous surface of gut [20]. This effect of Probiotics has been reported in various studies. Chandra (2002) studied the effects of Bacillus coagulans on severity of acute rotavirus diarrhea. The study was double-blind, randomized trial conducted in 112 healthy newborn term infants. These infants in rural India were administered with an oral dose of either $1 \times 10^{8}$ spores Bacillus coagulans $(\mathrm{n}=55)$ or placebo $(\mathrm{n}=57)$ for 12 months. Children were monitored for the episodes of rotavirus diarrhea during the course of study. Administration of Bacillus coagulans to infants decreased the episodes of rotavirus diarrhea and also reduced the duration of each episode with decrease in number of days of illness/year (13 days of illness in the Bacillus coagulans group as compared to 35 days in the control). No adverse effect was reported in the study [21]. Li in 2007 conducted uncontrolled trial in 47 patients (aged 46-81 years) with chronic diarrhea and abdominal distension. These patients were under the treatment of gentamycin, metronidazole, berberine and triperidol. These drugs were discontinued and Medilac-S containing Bacillus subtilis ( $3 \times 10^{9}$ $\mathrm{CFU} /$ day) was administered to patients for one month. Medilac-S was effective in treating the symptoms and there was no relapse. Patients also gained weight in varying degrees [22].

Wang et al in 2008 conducted a randomized control trial in 100 patients (aged 16-60 years) with chronic diarrhea to compare the efficacy of Bacillus subtilis + Smectite (phyllosilicate mineral) and Smectite alone. Patients were divided into two groups. Group one $(\mathrm{n}=50)$ received Bacillus subtilis $\left(3 \times 10^{9} \mathrm{CFU} / \mathrm{cap}\right)+$ Smectite and group two- control group $(n=50)$ received Smectite for 2 weeks. It was observed that combination was more effective than monotherapy with Smectite [23]. Hun L in 2009 evaluated the effect of Bacillus coagulans for improvement in abdominal pain and bloating in IBS patients. In this randomized, placebo-controlled trail, 44 subjects received either placebo or Bacillus coagulans GBI30 once daily for 8 weeks. Improvement in abdominal pain and bloating was seen in patients receiving Bacillus coagulans compared to placebo [24]. Majeed $\mathrm{M}$ et al in 2016 conducted a double blind randomized placebo controlled trial on Bacillus coagulans in 26 patients with Irritable Bowel Syndrome with Diarrhoea (IBS-D). The patients were divided into two groups, first group received Bacillus coagulans MTCC $5856\left(2 \times 10^{9} \mathrm{CFU} /\right.$ day) $(\mathrm{n}=18)$ and second group received placebo $(\mathrm{n}=18)$ for 90 days. Decrease in clinical symptoms like stool frequency, diarrhoea, abdominal pain, bloating was observed in group receiving Bacillus coagulans compared to placebo [25].

Cuentas AM et al in 2017 carried out the effect of Bacillus subtilis DE111 on 50 adults aged 18-65 years suffering from constipation and/or diarrhoea. They were administered either with Bacillus subtilis DE111 ( 1 x 109 CFU) or placebo for 90 days. Questionnaires were carried out on 1, 15, 45, 75, and 105 day and data was collected. Improvement was observed in Bacillus subtilis DE111 consuming group compared to placebo. Adverse effects were not observed [26].

\section{Conclusion}

Improvement in stool frequency and consistency along with pain in abdomen, sensation of bloating and flatulence were observed in both the probiotic groups. Data of patients in group A and group B was found to be statistically significant ( $p$ value $<$ 0.0001) compared to group C. It was also found that patients receiving Bacillus subtilis HU58 + Bacillus coagulans SC208 in combination experienced better relief in terms of stool frequency and consistency along with pain in abdomen, sensation of bloating and flatulence than the patients receiving Bacillus coagulans SC208 alone. It has been reported that the symptoms of pain in abdomen, diarrhea, sensation of bloating and flatulence have also been noted in the IBS-D cases. Hence, application of Bacillus coagulans SC208 in IBS-D can also be considered. Further these claims can be supported by the use of other strains of Bacillus coagulans in the IBS-D trails (vide supra). This comparison however needs to be studied in detail by conducting double blind randomized study in a larger sample size.

\section{Acknowledgement}

We thank Dr. Arun Arote for the laboratory evaluation of biochemical and organ function tests. We also thank Miss. Vidhya Prabhu for statistical analysis of the data. We acknowledge the inputs of Ms. Roda Dalal in the preparation of the manuscript of the published article. 


\section{References}

1. Barbut F, Meynard JL (2002) Managing antibiotic associated diarrhoea. BMJ 324: 1345-1346.

2. Elseviers MM, Van Camp Y, Nayaert S, Dure K, Annemans L, et al. (2015) Prevalence and management of antibiotic associated diarrhoea in general hospital. BMC Infect Dis 15: 129.

3. Issa I, Moucari R (2014) Probiotic for antibiotic-associated diarrhoea: do we have a verdict?. World of Gastroenterol 20: 17788-17795.

4. Mantegazza C, Molinari P, D Auria E, Sonnino M, Morelli L, et al. (2018) Probiotics and antibiotic-associated diarrhoea in children: A review and new evidence on Lactobacillus rhamnosus GG during and after antibiotic treatment. Pharmacol Res 128: 63-72.

5. Cremonini F, Di Caro S, Nista EC, Bartolozzi F, Capelli G, et al. (2002) Meta-analysis: the effect of probiotic administration on antibioticassociated diarrhoea. Aliment Pharmacol Ther 16: 1461-1467.

6. Perez C (2015) Probiotics for the treating acute diarrhea and preventing antibiotic-associated diarrhoea in children. Nutr Hosp 31: 64-67.

7. Hawrelak JA, Whitten DL, Myers SP (2005) Is Lactobacillus rhamnosus GG effective in preventing the onset of antibiotic-associated diarrhoea:a systematic review. Digestion 72: 51-56.

8. Hayes SR, Vargas AJ (2016) Probiotics for the Prevention of Pediatric Antibiotic-Associated Diarrhea. Explore (NY) 12: 463-466.

9. Blake MR, Rake JM, Whelan K (2016) Validity and reliability of the Bristol stool form scale in healthy adults and patients with diarrhoeapredominant irritable bowel syndrome. Aliment Pharmacol Ther 44: 693-703.

10. Wong S, Santullo P, O Driscoll J, Jamous A, Hirani SP, et al. (2017) Use of antibiotic and prevalence of antibiotic-associated diarrhoea in-patients with spinal cord injuries: a UK national spinal injury centre experience. Spinal cord 55: 583-587.

11. Cutting SM (2011) Bacillus probiotics. Food Microbiology 28(2): 214-20.

12. Hong HA, R Khaneja, NM Tam, A Cazzato, S Tan, et al. (2009a) Bacillus subtilis isolated from the human gastrointestinal tract. Res Microbiol 160(2): 134-143.

13. Hong HA, E To, S Fakhry, L Baccigalupi, E Ricca, et al. (2009b) Defining the natural habitat of Bacillus spore-formers. Res Microbiol 160(6): 375379.

\section{ISSN: 2574-1241}

DOI: $10.26717 /$ BJSTR.2020.29.004839

Dilip Mehta. Biomed J Sci \& Tech Res

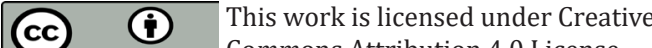

Commons Attribution 4.0 License

Submission Link: https://biomedres.us/submit-manuscript.php
14. Dound Y, Jadhav SS, Devale M, Bayne T, Krishnan K, et al. (2017) The effect of probiotic Bacillus subtilis HU58 on immune function in healthy human. The Indian practitioner 70: 15-20.

15. Mehta DS, De Souza A, Jadhav SS, Devale M (2020) A Study of Probiotic Bacillus subtilis HU58 for the Management of Antibiotic-Associated Diarrhoea in Adults. The Indian Practitioner 73(4): 22-28.

16. Lange K, Buerger M, Stallmach A, Bruns T (2016) Effects of antibiotics on gut microbiota. Dig Dis 34: 260-268.

17. Ha C, Lam YY, Holmes AJ (2014) Mechanistic links between gut microbial community dynamics, microbial functions and metabolic health. WJG 20: $16498-16517$

18. Morrison DJ, Preston T (2016) Formation of short chain fatty acids by the gut microbiota and their impact on human metabolism. Gut microbes 7: 189-200.

19. Suardi E, Crippa F, D’Arminio Monoforte A (2013) Probiotics in the prevention of antibiotic-associated diarrhea in adults. International Journal of Probiotics and Prebiotics 8: 41-44.

20. D'souza AL, Rajkumar C, Cooke J, Bulpitt CJ (2002) Probiotic in prevention of antibiotic associated diarrhoea: meta-analysis. BMJ 324: 1361.

21. Chandra PK (2002) Effect of Lactobacillus on the incidence and severity of acute rotavirus diarrhoea in infants. A prospective placebo-controlled double-blind study. Nutr Res 22: 65:69.

22. Lin Z (2007) Clinical observation of Medilac-S in the treatment of chronic diarrhoea. Medical Innovation Research 4: 100.

23. Wang YZ (2008) Clinical observation of combination of Medilac-S and Smecta in treatment of chronic diarrhoea. Chinese medicine of trauma and disability medicine 16: 70-71.

24. Hun L (2009) Bacillus coagulans significantly improved abdominal pain and bloating in patients with IBS. Postgrad Med 121: 119-124.

25. Majeed M, Nagabhushanam K, Nataranjan S, Sivakumar A, Ali F, et al. Bacillus coagulans MTCC 5856 supplementation in the management of diarrhoea predominant irritable bowel syndrome: a double blind randomized placebo controlled pilot clinical study. Nutr J 15: 21.

26. Cuetas AM, Deaton J, Khn S, Davidson J, Ardita C (2017) The effect of Bacillus subtilis DE111 on the daily bowel movement profile for people with occasional gastrointestinal irregularity. J Prob Health 5: 189.

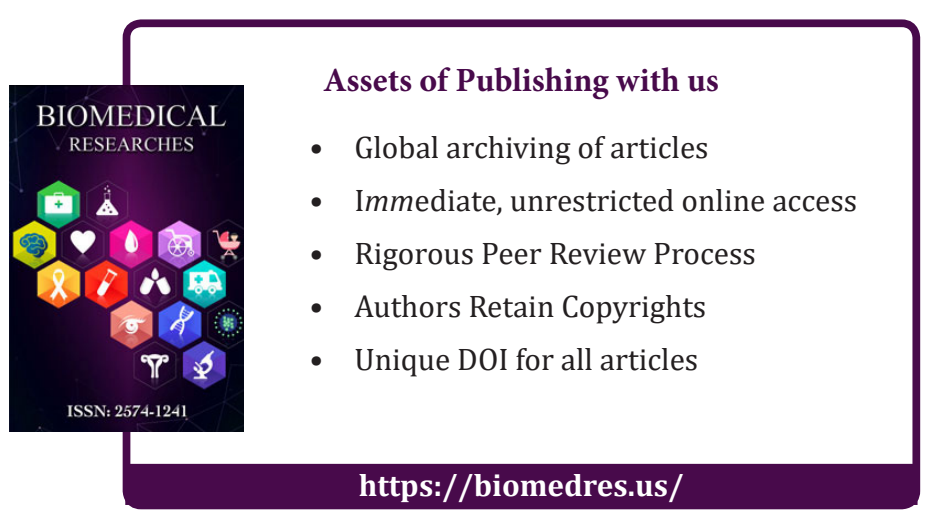

Copyright@ Dilip Mehta | Biomed J Sci \& Tech Res | BJSTR. MS.ID.004839. 